Homology, Homotopy and Applications, vol.12(2), 2010, pp.25-37

\title{
TORSION IN FINITE $H$-SPACES AND THE HOMOTOPY OF THE THREE-SPHERE
}

\author{
PIOTR BEBEN AND STEPHEN THERIAULT
}

(communicated by Donald M. Davis)

\begin{abstract}
Let $X$ be a 2-connected $p$-local finite $H$-space with a single cell in dimension three. We give a simple cohomological criterion which distinguishes when the inclusion $S^{3} \stackrel{i}{\longrightarrow} X$ has the property that the loop of its three-connected cover is null homotopic. In particular, such a null homotopy implies that $\pi_{m}(i)=0$ for $m \geqslant 4$. Applications are made to Harper's rank 2 finite $H$-space and simple, simply-connected, compact Lie groups.
\end{abstract}

\section{Introduction}

The existence of torsion in the cohomology of a finite $H$-space has been a subject of great interest for several decades (see, for example, $[\mathbf{H}, \mathbf{K}, \mathbf{L}]$ ). In this paper we show that, in certain situations, the existence of torsion in cohomology determines interesting homotopy theoretic information.

Assume that all spaces and maps have been localized at an odd prime $p$ and homology is taken with mod- $p$ coefficients. Let $X$ be a 2-connected finite $H$-space. Note that by $[\mathbf{L}]$, any simply-connected finite $H$-space is in fact 2-connected. Suppose that $X$ has a single cell in dimension three, $i: S^{3} \longrightarrow X$ is the inclusion of the bottom cell, and $x \in H^{3}(X)$ satisfies $i^{*}(x)=\iota$, where $\iota \in H^{3}\left(S^{3}\right)$ is a generator. We show that the cohomological property $\beta \mathcal{P}^{1}(x) \neq 0$ holds if and only if the loops on the threeconnected cover of $i$ is null homotopic. To phrase this another way, let $S^{3}\langle 3\rangle$ and $X\langle 3\rangle$ be the three-connected covers of $S^{3}$ and $X$ respectively, and let $i\langle 3\rangle$ be the three-connected cover of $i$. Then $\beta \mathcal{P}^{1}$ detects the triviality of $\Omega i\langle 3\rangle$.

These statements are intimately connected to the least nonvanishing torsion homotopy group of $S^{3}$. Let $\alpha: S^{2 p} \longrightarrow S^{3}$ be a representative of the generator of $\pi_{2 p}\left(S^{3}\right) \cong$ $\mathbb{Z} / p \mathbb{Z}$. Since $\alpha$ has order $p$, it extends to a map $\bar{\alpha}: P^{2 p+1}(p) \longrightarrow S^{3}$. Note that this extension is unique since the difference between any two choices of an extension factors through a map $S^{2 p+1} \longrightarrow S^{3}$, and $\pi_{2 p+1}\left(S^{3}\right)=0$. We show that, in general, if $X$ is any $H$-space and $i: S^{3} \longrightarrow X$ is any map then $\Omega i\langle 3\rangle$ is null homotopic if and only if $i \circ \bar{\alpha}$ is null homotopic. In the special case when $X$ is a simply-connected, finite $H$-space and $i$ is the inclusion of one of the bottom cells we relate a null homotopy

Received December 8, 2009, revised May 10, 2010; published on July 16, 2010.

2000 Mathematics Subject Classification: Primary 55P45, 55Q52.

Key words and phrases: finite $H$-space, Harper's space, torsion Lie group, three sphere.

This article is available at http://intlpress.com/HHA/v12/n2/a2

Copyright (C) 2010, International Press. Permission to copy for private use granted. 
for $i \circ \bar{\alpha}$ to the cohomological condition $\beta \mathcal{P}^{1}(x) \neq 0$. When $X$ has a single cell in dimension 3, the null homotopy for $i \circ \bar{\alpha}$ becomes equivalent to having $\beta \mathcal{P}^{1}(x) \neq 0$.

Theorem 1.1. Let $X$ be an $H$-space and $i: S^{3} \longrightarrow X$ be a map. The following are equivalent:

(a) the composite $P^{2 p+1}(p) \stackrel{\bar{\alpha}}{\longrightarrow} S^{3} \stackrel{i}{\longrightarrow} X$ is null homotopic;

(b) the map $\Omega S^{3}\langle 3\rangle \stackrel{\Omega i\langle 3\rangle}{\longrightarrow} \Omega X\langle 3\rangle$ is null homotopic.

If $X$ is a 2-connected finite $H$-space with a single cell in dimension three, $i$ is the inclusion of the bottom cell, and $x \in H^{3}(X)$ satisfies $i^{*}(x)=\iota$, then parts (a) and (b) are also equivalent to:

(c) $\beta \mathcal{P}^{1}(x) \neq 0$.

In Section 3 we consider the more general case of a 2-connected finite $H$-space $X$ with multiple cells in dimension three, and give a condition for when a map $S^{3} \stackrel{i}{\longrightarrow} X$ satisfying $i^{*}(x)=\iota$ and $\beta \mathcal{P}^{1}(x) \neq 0$ has the property that $\Omega i\langle 3\rangle$ is null homotopic. However, the statement is too technical to reasonably describe at this point.

From Theorem 1.1 (b) we immediately obtain the following corollary.

Corollary 1.2. The map $S^{3} \stackrel{i}{\longrightarrow} X$ has the property that $\pi_{m}(i)=0$ for $m \geqslant 4$.

The implication (a) implies (b) in Theorem 1.1 is related to a deep conjecture in homotopy theory, that $\Omega^{2} \bar{\alpha}\langle 3\rangle$ has a left homotopy inverse. If this were the case, then (a) would imply that the map $\Omega^{2} S^{3}\langle 3\rangle \stackrel{\Omega^{2} i\langle 3\rangle}{\longrightarrow} \Omega^{2} X\langle 3\rangle$ is null homotopic, with no condition on $X$. In this sense the $H$-space hypothesis on $X$ in Theorem 1.1 should not be regarded as best possible. Rather, it allows us to go forward and suffices for the applications we have in mind.

We give two useful examples. First, Harper $[\mathbf{H}]$ constructed a rank 2 mod- $p$ finite $H$-space $K_{p}$ which is analogous to the Lie group $G_{2}$ at the prime 2 , in the sense that

$$
H^{*}\left(K_{p}\right)=\Lambda\left(x_{3}, y_{2 p+1}\right) \otimes \mathbb{Z} / p \mathbb{Z}\left[z_{2 p+2}\right] /\left(z_{2 p+2}^{p}\right)
$$

with $\mathcal{P}^{1}(x)=y$ and $\beta(y)=z$. Since $\beta \mathcal{P}^{1}(x) \neq 0$, Theorem 1.1 implies that the map $\Omega S^{3}\langle 3\rangle \stackrel{\Omega i\langle 3\rangle}{\longrightarrow} \Omega K_{p}\langle 3\rangle$ is null homotopic, and so $\pi_{m}(i)=0$ for $m \geqslant 4$. Second, a torsion Lie group is a Lie group which has $p$-torsion in its integral cohomology. When $p$ is odd the only simple, simply-connected, compact torsion Lie groups are $F_{4}, E_{6}, E_{7}$, and $E_{8}$ at the prime 3 , and $E_{8}$ at the prime 5 . In each case, there is a single generator $x$ in degree three cohomology and $\beta \mathcal{P}^{1}(x) \neq 0$. On the other hand, if $G$ is a simple, simply-connected, compact Lie group which is torsion free then there is a single generator $x \in H^{3}(G)$ and the fact that $G$ is torsion free implies that $\beta \mathcal{P}^{1}(x)=0$. Hence Theorem 1.1 implies the following.

Theorem 1.3. Let $p \geqslant 3$ and let $G$ be a simple, simply-connected, compact Lie group. The inclusion $S^{3} \stackrel{i}{\longrightarrow} G$ has the property that $\Omega S^{3}\langle 3\rangle \stackrel{\Omega i\langle 3\rangle}{\longrightarrow} \Omega G\langle 3\rangle$ is null homotopic if and only if $G$ is a torsion Lie group. 
In proving Theorem 1.1 we use an $H$-space $B(3,2 p+1)$ studied by Toda [To1], which has three key properties. First,

$$
H^{*}(B(3,2 p+1)) \cong \Lambda(x, y)
$$

where $|x|=3,|y|=2 p+1$ and $\mathcal{P}^{1}(x)=y$. Second, there is a homotopy fibration

$$
S^{3} \stackrel{s}{\longrightarrow} B(3,2 p+1) \stackrel{q}{\longrightarrow} S^{2 p+1}
$$

where $q^{*}$ is an injection and $s^{*}$ is a projection. Third, there is a "characteristic map"

$$
c: S^{2 p+1} \longrightarrow B(3,2 p+1)
$$

with the property that $q \circ c$ is of degree $p$. We show that the map $S^{3} \stackrel{i}{\longrightarrow} X$ extends to a map $j: B(3,2 p+1) \longrightarrow X$ with the property that $j \circ c$ is null homotopic. In Proposition 4.1 we show that if $p \geqslant 5$ then the $p^{t h}$-power map

$$
\Omega B(3,2 p+1)\langle 3\rangle \stackrel{p}{\longrightarrow} \Omega B(3,2 p+1)\langle 3\rangle
$$

factors through $\Omega c$. This is then used to prove the following (the $p=3$ case arises from different methods).

Theorem 1.4. Let $X$ be a finite $H$-space as in Theorem 1.1. If $p \geqslant 5$, the map

$$
\Omega B(3,2 p+1)\langle 3\rangle \stackrel{\Omega j\langle 3\rangle}{\longrightarrow} \Omega X\langle 3\rangle
$$

has order $p$. If $p=3$, the map

$$
\Omega^{2} B(3,7)\langle 3\rangle \stackrel{\Omega^{2} j\langle 3\rangle}{\longrightarrow} \Omega^{2} X\langle 3\rangle
$$

has order 3 .

In particular, the map $\Omega B(3,2 p+1)\langle 3\rangle \stackrel{\Omega j\langle 3\rangle}{\longrightarrow} \Omega K_{p}\langle 3\rangle$ has order $p$; if $G$ is one of $F_{4}, E_{6}, E_{7}$, or $E_{8}$, then localized at 3 the map $\Omega B(3,7)\langle 3\rangle \stackrel{\Omega j\langle 3\rangle}{\longrightarrow} \Omega G\langle 3\rangle$ has order 3 ; and localized at 5 the map $\Omega B(3,11)\langle 3\rangle \stackrel{\Omega j\langle 3\rangle}{\longrightarrow} \Omega E_{8}\langle 3\rangle$ has order 5 . For the latter case, it is interesting to note that by $[\mathbf{M N T}]$, there is a 5-local homotopy equivalence $e: B(3,11) \longrightarrow G_{2}$. Since $E_{8}$ at 5 is the Eilenberg-MacLane space $K(\mathbb{Z}, 3)$ in dimensions $\leqslant 14$ and both $G_{2}$ and $B(3,11)$ are of dimension 14 , the homotopy classes of the standard inclusion $\imath: G_{2} \longrightarrow E_{8}$ and the map $B(3,11) \stackrel{j}{\longrightarrow} E_{8}$ are determined by $H^{3}\left(G_{2} ; \mathbb{Z} / 5 \mathbb{Z}\right)$ and $H^{3}(B(3,11) ; \mathbb{Z} / 5 \mathbb{Z})$ respectively. Since $e$ can be chosen to be the identity on $H^{3}$, we obtain $e \circ i \simeq j$. Thus $\Omega i\langle 3\rangle$ has order 5 .

\section{The equivalence of parts (a) and (b) of Theorem 1.1}

The difficult implication is (b) implies (a). To prepare for this, we need the following initial construction.

Proposition 2.1. Let $X$ be an $H$-space and $i: S^{3} \longrightarrow X$ be any map. Suppose that the composition $P^{2 p+1}(p) \stackrel{\bar{\alpha}}{\longrightarrow} S^{3} \stackrel{i}{\longrightarrow} X$ is null homotopic. Then $i$ extends to a map $j: B(3,2 p+1) \longrightarrow X$ which can be chosen so that the composite

$$
S^{2 p+1} \stackrel{c}{\longrightarrow} B(3,2 p+1) \stackrel{j}{\longrightarrow} X
$$

is null homotopic. 
Proposition 2.1 requires three preliminary steps. First, define the space $\bar{A}$ by the homotopy cofibration

$$
P^{2 p+1}(p) \stackrel{\bar{\alpha}}{\longrightarrow} S^{3} \stackrel{i}{\longrightarrow} \bar{A} .
$$

As a module over the Steenrod algebra we have $H^{*}(\bar{A}) \cong\left\{y, \mathcal{P}^{1}(y), \beta \mathcal{P}^{1}(y)\right\}$, where $|y|=3$. Observe also that $\bar{A}$ is the $(2 p+2)$-skeleton of $K(\mathbb{Z}, 3)$. Since one of our assumptions on $X$ is that the composition $P^{2 p+1}(p) \stackrel{\bar{\alpha}}{\longrightarrow} S^{3} \stackrel{i}{\longrightarrow} X$ is null homotopic, the following lemma is an immediate consequence.

Lemma 2.2. There is an extension

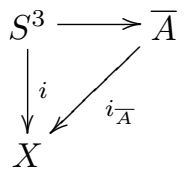

for some map $i_{\bar{A}}$.

Second, let $A$ be the $(2 p+1)$-skeleton of $\bar{A}$. As a module over the Steenrod algebra we have $H^{*}(A) \cong\left\{x, \mathcal{P}^{1}(x)\right\}$. In particular, observe that $A$ is also the $(2 p+1)$ skeleton of $B(3,2 p+1)$. Consider the cofibration

$$
S^{2 p+1} \stackrel{d}{\longrightarrow} A \longrightarrow \bar{A}
$$

where $d$ attaches the top cell to $\bar{A}$.

Lemma 2.3. The following hold:

(a) the map $d$ represents a generator of $\pi_{2 p+1}(A) \cong \mathbb{Z}$;

(b) the composite $S^{2 p+1} \stackrel{d}{\longrightarrow} A \longrightarrow B(3,2 p+1)$ is homotopic to $\pm c$.

Proof. Consider the homotopy fibration $F \longrightarrow A \longrightarrow \bar{A}$, which defines the space $F$. It was observed that $\bar{A}$ is the $(2 p+2)$-skeleton of $K(\mathbb{Z}, 3)$. However, the next cell in $K(\mathbb{Z}, 3)$ occurs in dimension $2 p+4$, as the product of the degree 3 and $(2 p+1)$ cells. Thus $\bar{A}$ is actually the $(2 p+3)$-skeleton of $K(\mathbb{Z}, 3)$. In other words, the map $\bar{A} \longrightarrow K(\mathbb{Z}, 3)$ is $(2 p+2)$-connected, implying that $\pi_{m}(A)=0$ for $3<m<2 p+2$. Thus the map $F \longrightarrow A$ induces an isomorphism on $\pi_{2 p+1}$. On the other hand, a Serre spectral sequence calculation shows that the $(2 p+3)$-skeleton of $F$ is $S^{2 p+1}$. Thus $\pi_{2 p+1}(F)=\pi_{2 p+1}\left(S^{2 p+1}\right) \cong \mathbb{Z}$, and therefore $\pi_{2 p+1}(A) \cong \mathbb{Z}$. Further, the composite $S^{2 p+1} \hookrightarrow F \longrightarrow A$ is homotopic to the attaching map $d$, implying that $d$ represents a generator of $\pi_{2 p+1}(A)$, proving part (a).

Next, observe that the skeletal inclusion $A \longrightarrow B(3,2 p+1)$ is $(2 p+2)$-connected. Thus it induces an isomorphism on $\pi_{2 p+1}$. By part (a), $d$ represents a generator of $\pi_{2 p+1}(A) \cong \mathbb{Z}$ while by [To1], $c$ represents a generator of $\pi_{2 p+1}(B(3,2 p+1)) \cong \mathbb{Z}$. Part (b) now follows immediately.

For the third preliminary step, let $i_{A}$ be the composite $i_{A}: A \longrightarrow \bar{A} \stackrel{i_{\bar{A}}}{\longrightarrow} X$, where the left map is the skeletal inclusion. Observe that as $i_{\bar{A}}$ extends the map $S^{3} \stackrel{i}{\longrightarrow} X$, so does $i_{A}$. 
Lemma 2.4. The map $A \stackrel{i_{A}}{\longrightarrow} X$ extends to a map $j: B(3,2 p+1) \longrightarrow X$. Consequently, $j$ also extends $i$.

Proof. Observe that there is a homotopy cofibration $S^{2 p+3} \stackrel{\theta}{\longrightarrow} A \longrightarrow B(3,2 p+1)$ where $\theta$ attaches the top cell to $B(3,2 p+1)$. To show that $i_{A}$ extends to $j$ we need to show that the composite $i_{A} \circ \theta$ is null homotopic. Since $X$ is an $H$-space, it is equivalent to show that $\Sigma i_{A} \circ \Sigma \theta$ is null homotopic. We claim that this is true because $\Sigma \theta$ itself is null homotopic. This is a consequence of the following general result of $[\mathbf{C N}]$. Let $Y$ be an $H$-space with $H_{*}(Y) \cong \Lambda(y, z)$, where $|y|=2 m+1$ and $|z|=2 n+1$. Without loss of generality, suppose that $n \geqslant m$. Let $Z$ be the $(2 n+1)$-skeleton of $Y$, so $H_{*}(Z) \cong\{x, y\}$. Then there is a homotopy equivalence $\Sigma Y \simeq \Sigma Z \vee \Sigma S^{2 n+2 m+2}$. In particular, the map $S^{2 n+2 m+1} \longrightarrow Z$ attaching the top cell to $Y$ is null homotopic when suspended.

Proof of Proposition 2.1: By Lemma 2.4, the map $j$ extends $i$. To show that $c \circ j$ is null homotopic, consider the diagram

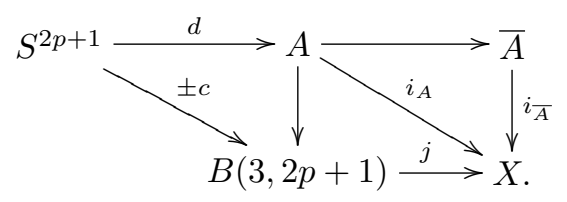

The left triangle homotopy commutes by Lemma 2.3 (b). The upper right triangle homotopy commutes by definition of $i_{A}$ as the skeletal restriction of $i_{\bar{A}}$. The lower right triangle homotopy commutes by Lemma 2.4. Since the top row is two consecutive maps in a cofibration, it is null homotopic. The homotopy commtutativity of the diagram as a whole therefore implies that $j \circ c$ is null homotopic.

Next, we aim towards Corollary 2.6 which describes a crucial property of the map $S^{2 p+1} \stackrel{c}{\longrightarrow} B(3,2 p+1)$. Define the space $S^{2 p+1}\{p\}$ and the map $\delta$ by the homotopy fibration

$$
S^{2 p+1}\{p\} \stackrel{\delta}{\longrightarrow} S^{2 p+1} \stackrel{p}{\longrightarrow} S^{2 p+1} .
$$

A Serre spectral sequence calculation directly shows that

$$
H^{*}\left(S^{2 p+1}\{p\}\right) \cong \Lambda\left(x_{2 p+1}\right) \otimes \Gamma\left[y_{2 p}\right]
$$

with $\beta(x)=y$. In particular, the bottom cell of $S^{2 p+1}\{p\}$ is in dimension $2 p$.

As mentioned in the introduction, the composite

$$
S^{2 p+1} \stackrel{c}{\longrightarrow} B(3,2 p+1)\langle 3\rangle \stackrel{q\langle 3\rangle}{\longrightarrow} S^{2 p+1}
$$

is of degree $p$. From this we obtain a homotopy pullback diagram

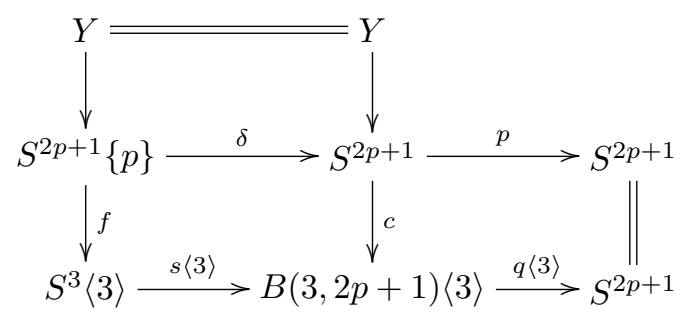


which defines the space $Y$ and the map $f$. By [To1], the space $Y$ is $\left(2 p^{2}-2\right)$ connected.

Lemma 2.5. The map $\Omega S^{2 p+1}\{p\} \stackrel{\Omega f}{\longrightarrow} \Omega S^{3}\langle 3\rangle$ has a right homotopy inverse.

Proof. It is well known that there are isomorphisms

$$
H_{*}\left(\Omega S^{2 p+1}\{p\}\right) \cong \otimes_{j=1}^{\infty}\left(\Lambda\left(a_{2 p^{j}-1}\right) \otimes \mathbb{Z} / p \mathbb{Z}\left[b_{2 p^{j}-2}\right]\right)
$$

and

$$
H_{*}\left(\Omega S^{3}\langle 3\rangle\right) \cong \Lambda\left(x_{2 p-1}\right) \otimes \mathbb{Z} / p \mathbb{Z}\left[y_{2 p-2}\right] .
$$

Since the homotopy fibre $\Omega Y$ of $\Omega f$ is $\left(2 p^{2}-3\right)$-connected, $\Omega f$ is a homotopy equivalence when restricted to $2 p$-skeleta. Therefore, for degree reasons, we have

$$
(\Omega f)_{*}\left(a_{2 p-1}\right)=x_{2 p-1}
$$

and

$$
(\Omega f)_{*}\left(b_{2 p-2}\right)=y_{2 p-2} .
$$

Since $(\Omega f)_{*}$ is an algebra map, the composite

$$
\Lambda\left(a_{2 p-1}\right) \otimes \mathbb{Z} / p \mathbb{Z}\left[b_{2 p-2}\right] \hookrightarrow H_{*}\left(\Omega S^{2 p+1}\{p\}\right) \stackrel{(\Omega f)_{*}}{\longrightarrow} H_{*}\left(\Omega S^{3}\langle 3\rangle\right)
$$

is therefore the identity map.

By [GraT], for each $p \geqslant 3$, there is a space $T^{2 p+1}$ and a map $T^{2 p+1} \stackrel{g}{\longrightarrow} \Omega S^{2 p+1}\{p\}$ which geometrically realizes the inclusion

$$
\Lambda\left(a_{2 p-1}\right) \otimes \mathbb{Z} / p \mathbb{Z}\left[b_{2 p-2}\right] \hookrightarrow H_{*}\left(\Omega S^{2 p+1}\{p\}\right) .
$$

Thus the composite $T^{2 p+1} \stackrel{g}{\longrightarrow} \Omega S^{2 p+1}\{p\} \stackrel{\Omega f}{\longrightarrow} \Omega S^{3}\langle 3\rangle$ induces the identity map in homology and so is a homotopy equivalence. Hence $\Omega f$ has a right homotopy inverse.

Let $t: \Omega S^{3}\langle 3\rangle \longrightarrow \Omega S^{2 p+1}\{p\}$ be a right homotopy inverse of $\Omega f$. Looping the lower left square in (1) and precomposing with $t$, we obtain the following.

Corollary 2.6. There is a homotopy commutative diagram

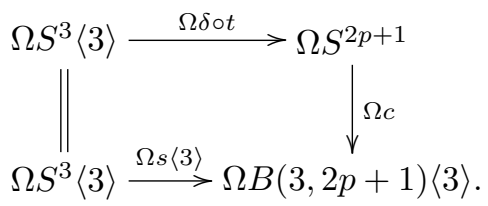

In other words, Corollary 2.6 states that $\Omega j\langle 3\rangle$ lifts through $\Omega c$, and $\Omega \delta \circ t$ is a specific choice of a lift.

Proof of the equivalence of parts (a) and (b) in Theorem 1.1. Suppose that $i \circ \bar{\alpha}$ is null homotopic. By Proposition 2.1, $i$ extends to a map $B(3,2 p+1) \stackrel{j}{\longrightarrow} X$ with 
the property that $j \circ c$ is null homotopic. Note that by connectivity, the composite $S^{3} \stackrel{s}{\longrightarrow} B(3,2 p+1) \stackrel{j}{\longrightarrow} X$ is homotopic to $i$. Consider the diagram

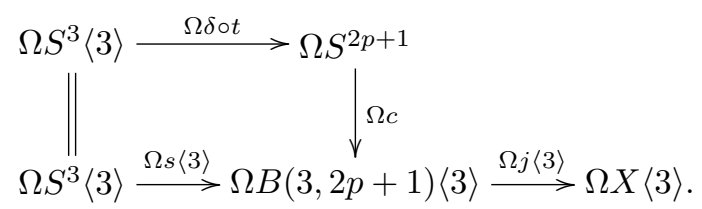

The square homotopy commutes by Corollary 2.6. Since $j \circ c$ is null homotopic, the upper direction around the diagram is null homotopic. Thus the lower direction direction around the diagram - the composite $\Omega j\langle 3\rangle \circ \Omega s\langle 3\rangle$ - is null homotopic. Therefore, as $j \circ s \simeq i$, the map $\Omega i\langle 3\rangle$ is null homotopic.

Conversely, suppose that $\Omega S^{3}\langle 3\rangle \stackrel{\Omega i\langle 3\rangle}{\longrightarrow} \Omega X\langle 3\rangle$ is null homotopic. To show that the composite $P^{2 p+1}(p) \stackrel{\bar{\alpha}}{\longrightarrow} S^{3} \stackrel{i}{\longrightarrow} X$ is null homotopic, it is equivalent to adjoint and show that the composite $P^{2 p}(p) \longrightarrow \Omega S^{3} \stackrel{\Omega i}{\longrightarrow} \Omega X$ is null homotopic. Since $\bar{\alpha}$ factors through $S^{3}\langle 3\rangle$, the adjoint of $i \circ \bar{\alpha}$ factors through the map $\Omega S^{3}\langle 3\rangle \stackrel{\Omega i\langle 3\rangle}{\longrightarrow} \Omega X\langle 3\rangle$, implying that it is null homotopic.

\section{Finite $H$-spaces and part (c) of Theorem $\mathbf{1 . 1}$}

Now assume that $X$ is a 2-connected finite $H$-space. We will show that parts (a) and (c) of Theorem 1.1 are equivalent. The easy implication is (a) implies (c), which we prove in greater generality.

Lemma 3.1. Let $Y$ be a space and suppose there is a map $f: S^{3} \longrightarrow Y$ such that $f^{*}(x)=\iota$ for some $x \in H^{3}(Y)$. If the composite $P^{2 p+1}(p) \stackrel{\bar{\alpha}}{\longrightarrow} S^{3} \stackrel{f}{\longrightarrow} Y$ is null homotopic then $\beta \mathcal{P}^{1}(x) \neq 0$.

Proof. The null homotopy for $f \circ \bar{\alpha}$ implies that there is an extension

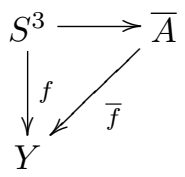

for some map $\bar{f}$. Recall that $H^{*}(\bar{A}) \cong\left\{y, \mathcal{P}^{1}(y), \beta \mathcal{P}^{1}(y)\right\}$, where $|y|=3$. As $f^{*}(x)=\iota$, the homotopy commutativity of this diagram implies that $\bar{f}^{*}(x)=y$. The naturality of the Steenrod operations then imply that $\bar{f}^{*}\left(\beta \mathcal{P}^{1}(x)\right)=\beta \mathcal{P}^{1}\left(\bar{f}^{*}(x)\right)=\beta \mathcal{P}^{1}(y)$. But $\beta \mathcal{P}^{1}(y) \neq 0$ in $H^{*}(A)$. Therefore $\beta \mathcal{P}^{1}(x) \neq 0$ in $H^{*}(Y)$.

Now we turn to the difficult implication, (c) implies (a). We do this in greater generality than is stated in Theorem 1.1 by considering any 2-connected finite $H$ space $X$, possibly with many cells in dimension 3 , and any map $S^{3} \stackrel{i}{\longrightarrow} X$ such that $i^{*}(x)=\iota$ for some $x \in H^{3}(X)$ with $\beta \mathcal{P}^{1}(x) \neq 0$. We give a condition for when $i \circ \bar{\alpha}$ is null homotopic. In the special case when $X$ has a single cell in dimension 3 and $i$ is the inclusion of the bottom cell, the condition will be automatically satisfied. 
We first establish a homotopy equivalence for the $(2 p+2)$-skeleton of $X$, which is stated in Proposition 3.2. We begin with some linear algebra designed to find a basis of $H^{3}(X)$ which is well behaved with respect to the Steenrod operations $\mathcal{P}^{1}$ and $\beta \mathcal{P}^{1}$.

By hypothesis, the map $S^{3} \stackrel{i}{\longrightarrow} X$ has the property that there is an $x \in H^{3}(X)$ such that $i^{*}(x)=\iota$ and $\beta \mathcal{P}^{1}(x) \neq 0$. Let $y_{1}=x$, and extend to a basis $\left\{y_{1}, \ldots, y_{r}\right\}$ of $H^{3}(X)$. Consider the set $\left\{\beta \mathcal{P}^{1}\left(y_{1}\right), \ldots, \beta \mathcal{P}^{1}\left(y_{r}\right)\right\}$. Some of these elements are nonzero, some are zero, and some may be linear combinations of others. Reordering if necessary, assume that $\beta \mathcal{P}^{1}\left(y_{i}\right) \neq 0$ for $1 \leqslant i \leqslant r_{1}$, and $\beta \mathcal{P}^{1}\left(y_{i}\right)=0$ for $r_{1}<i \leqslant r$. In the case $1 \leqslant i \leqslant r_{1}$, reordering again if necessary, assume that the set $S=\left\{\beta \mathcal{P}^{1}\left(y_{i}\right)\right\}_{i=1}^{k}$ consists of a maximal number of linearly independent elements, while the set $T=$ $\left\{\beta \mathcal{P}^{1}\left(y_{i}\right)_{i=k+1}^{r_{1}}\right\}$ consists of elements which are linear combinations of elements in $S$. So for $\beta \mathcal{P}^{1}\left(y_{i}\right) \in T$, we have $\beta \mathcal{P}^{1}\left(y_{i}\right)=\Sigma_{j=1}^{t} u_{j_{i}} \beta \mathcal{P}^{1}\left(y_{j_{i}}\right)$ where $t \leqslant k$ and each $u_{j_{i}}$ is a unit in $\mathbb{Z} / p \mathbb{Z}$. Let $\bar{y}_{i}=y_{i}-\Sigma_{j=1}^{t} u_{j_{i}} y_{j_{i}}$. Then $\beta \mathcal{P}^{1}\left(y_{i}\right)=0$. Relabel $\bar{y}_{i}$ as $y_{i}$. Doing this for each $k+1 \leqslant i \leqslant r_{1}$, we obtain a new basis $\left\{y_{1}, \ldots, y_{r}\right\}$ for $H^{3}(X)$ which has the property that the set $\left\{\beta \mathcal{P}^{1}\left(y_{i}\right)\right\}_{i=1}^{k}$ is linearly independent while $\beta \mathcal{P}^{1}\left(y_{i}\right)=0$ for $k<i \leqslant r$.

Now consider the set $\left\{\mathcal{P}^{1}\left(y_{i}\right)\right\}_{i=1}^{r}$. Observe that as the set $\left\{\beta \mathcal{P}^{1}\left(y_{i}\right)\right\}_{i=1}^{k}$ is linearly independent so is the set $\bar{S}=\left\{\mathcal{P}^{1}\left(y_{i}\right)\right\}_{i=1}^{k}$. As for the remaining elements, $U=\left\{\mathcal{P}^{1}\left(y_{i}\right)\right\}_{i=k+1}^{r}$, some are nonzero, some are zero, and some may be linear combinations of elements in $\bar{S}$ and other elements in $U$. Arguing as before, we can alter the basis $\left\{y_{1}, \ldots, y_{r}\right\}$ of $H^{3}(X)$ so that: (i) $\left\{y_{1}, \ldots, y_{k}\right\}$ has remained fixed, and (ii) the set $\left\{\mathcal{P}^{1}\left(y_{i}\right)\right\}_{i=1}^{l}$ is linearly independent, where $l \geqslant k$, while $\mathcal{P}^{1}\left(y_{i}\right)=0$ for $l<i \leqslant r$.

The extra condition we consider is:

(*) Suppose that this basis $\mathcal{B}=\left\{y_{1}, \ldots, y_{r}\right\}$ of $H^{3}(X)$ can be chosen such that $i^{*}\left(y_{i}\right)=0$ for $2 \leqslant i \leqslant r$.

Observe that condition $(*)$ is automatically satisfied if $X$ has a single cell in dimension 3 .

For a space $Y$, let $Y_{m}$ be the $m$-skeleton of $Y$.

Proposition 3.2. Let $X$ be a 2-connected finite $H$-space. Then there is a homotopy equivalence

$$
X_{2 p+2} \simeq\left(\prod_{j=1}^{k} \bar{A} \times \prod_{j=k+1}^{l} A \times \prod_{j=l+1}^{m} S^{2 i_{j}+1}\right)_{2 p+2}
$$

where $3 \leqslant 2 i_{j}+1 \leqslant 2 p+1$ for each $l+1 \leqslant i_{j} \leqslant m$. Further, if the basis $\mathcal{B}$ for $H^{3}(X)$ satisfies condition $(*)$ then this homotopy equivalence has the property that the map $S^{3} \stackrel{i}{\longrightarrow} X_{2 p+2}$ factors as the composite $S^{3} \hookrightarrow \bar{A}_{1} \hookrightarrow X_{2 p+2}$.

Proof. The fact that $X$ is a 2-connected finite $H$-space imposes many restrictions on the structure of $H^{*}(X)$. In particular, by $[\mathbf{L}]$, the least degree even dimensional generator can occur in degree $2 p+2$, and such a generator $z \in H^{2 p+2}(X)$ must be of the form $z=\beta \mathcal{P}^{1}(w)$ for some $w \in H^{3}(X)$. Consequently, in dimensions $\leqslant 2 p+2$, $H^{*}(X)$ is the direct sum of an exterior algebra on odd degree generators and the generating set of $H^{2 p+2}(X)$, with the latter consisting of elements of the form $\beta \mathcal{P}^{1}(w)$. 
Using the chosen basis of $H^{3}(X)$ we can write this more explicitly as

$$
\begin{array}{r}
H^{*}(X) \cong\left(\otimes_{i=1}^{k}\left[\Lambda\left(y_{i}, \mathcal{P}^{1}\left(y_{i}\right)\right) \oplus \beta \mathcal{P}^{1}\left(y_{i}\right)\right]\right) \otimes\left(\otimes_{i=k+1}^{l} \Lambda\left(y_{i}, \mathcal{P}^{1}\left(y_{i}\right)\right)\right) \\
\otimes \Lambda\left(y_{l+1}, \ldots, y_{r}, x_{2 i_{r+1}+1}, \ldots, x_{2 i_{m}+1}\right)
\end{array}
$$

where $\left|x_{2 i_{j}+1}\right|=2 i_{j}+1$ for $3 \leqslant 2 i_{j}+1 \leqslant 2 p+1$.

Each generator $y_{i} \in H^{3}\left(X_{2 p+2}\right)$ is represented by a map $\epsilon_{i}: X_{2 p+2} \longrightarrow K(\mathbb{Z}, 3)$. For dimensional reasons, $\epsilon_{i}$ factors through the $(2 p+2)$-skeleton of $K(\mathbb{Z}, 3)$, which is homotopy equivalent to $\bar{A}$. So $\epsilon_{i}$ induces a map $\bar{\epsilon}_{i}: X_{2 p+2} \longrightarrow \bar{A}$. In particular, if $1 \leqslant i \leqslant k$, then the product of the maps $\bar{\epsilon}_{i}$,

$$
X_{2 p+2} \longrightarrow \prod_{i=1}^{k} \bar{A}
$$

induces an injection onto the submodule $\otimes_{i=1}^{k}\left[\Lambda\left(y_{i}, \mathcal{P}^{1}\left(y_{i}\right)\right) \oplus \beta \mathcal{P}^{1}\left(y_{i}\right)\right]$ of $H^{*}\left(X_{2 p+2}\right)$. If $k<i \leqslant l$ then the fact that $\mathcal{P}^{1}\left(y_{i}\right) \neq 0$ but $\beta \mathcal{P}^{1}\left(i_{i}\right)=0$ implies that the composite $X_{2 p+2} \stackrel{\bar{\epsilon}_{i}}{\longrightarrow} \bar{A} \stackrel{q}{\longrightarrow} S^{2 p+2}$ is null homotopic, where $q$ is the pinch map to the top cell. Thus $\bar{\epsilon}_{i}$ lifts to the homotopy fibre $F$ of $q$. A Serre spectral sequence calculation immediately shows that $H^{*}\left(F_{2 p+2}\right) \cong H^{*}(A)$, implying that $F_{2 p+2} \simeq A$. Thus the lift of $\bar{\epsilon}_{i}$ induces a map $\varepsilon_{i}: X_{2 p+2} \longrightarrow A$, and the product of the maps $\varepsilon_{i}$,

$$
X_{2 p+2} \longrightarrow \prod_{i=k+1}^{l} A
$$

induces an injection onto the submodule $\otimes_{i=k+1}^{l} \Lambda\left(y_{i}, \mathcal{P}^{1}\left(y_{i}\right)\right)$ of $H^{*}\left(X_{2 p+2}\right)$. If $l<i \leqslant$ $r$, then the fact that $\mathcal{P}^{1}\left(y_{i}\right)=0$ implies that the composite $X_{2 p+2} \stackrel{\bar{\epsilon}_{i}}{\longrightarrow} \bar{A} \stackrel{\bar{q}}{\longrightarrow} P^{2 p+2}(p)$ is null homotopic, where $\bar{q}$ is the pinch map to the top Moore space. Thus $\bar{\epsilon}_{i}$ lifts to the homotopy fibre $\bar{F}$ of $\bar{q}$. A Serre spectral sequence calculation immediately shows that $H^{*}\left(\bar{F}_{2 p+2}\right) \cong H^{*}\left(S^{3}\right)$, implying that $\bar{F}_{2 p+2} \simeq S^{3}$. Thus the lift of $\bar{\epsilon}_{i}$ induces a map $\varepsilon_{i}: X_{2 p+2} \longrightarrow S^{3}$, and the product of the maps $\varepsilon_{i}$,

$$
X_{2 p+2} \longrightarrow \prod_{i=l+1}^{r} S^{3}
$$

induces an injection onto the submodule $\Lambda\left(y_{l+1}, \ldots, y_{r}\right)$ of $H^{*}\left(X_{2 p+2}\right)$.

Next, each generator $x_{2 i_{j}+1} \in H^{*}\left(X_{2 p+2}\right)$ is represented by a map

$$
\epsilon_{i_{j}}: X_{2 p+2} \longrightarrow K\left(\mathbb{Z}, 2 i_{j}+1\right) .
$$

For dimensional reasons, $\epsilon_{i_{j}}$ factors through the $(2 p+2)$-skeleton of $K\left(\mathbb{Z}, 2 i_{j+1}\right)$, which is homotopy equivalent to $S^{2 i_{j}+1}$ because $2 i_{j}+1 \geqslant 5$. Thus $\epsilon_{i_{j}}$ induces a map $\bar{\epsilon}_{i_{j}}: X_{2 p+2} \longrightarrow S^{2 i_{j}+1}$. Taking the product of the maps $\bar{\epsilon}_{i_{j}}$ for $r<j \leqslant m$ gives a map

$$
X_{2 p+2} \longrightarrow \prod_{j=r+1}^{m} S^{2 i_{j}+1}
$$

which induces an injection onto the submodule $\Lambda\left(x_{2 i_{r+1}+1}, \ldots, x_{2 i_{m}+1}\right)$ of $H^{*}\left(X_{2 p+2}\right)$. 
Collectively, we obtain a product map

$$
X_{2 p+2} \longrightarrow\left(\prod_{j=1}^{k} \bar{A} \times \prod_{j=k+1}^{l} A \times \prod_{j=l+1}^{m} S^{2 i_{j}+1}\right)_{2 p+2}
$$

which induces an isomorphism in cohomology, implying that it is a homotopy equivalence.

Finally, suppose the basis $\mathcal{B}$ for $H^{3}(X)$ satisfies condition $(*)$. Since the homotopy equivalence for $X_{2 p+2}$ was determined by mapping into Eilenberg-Mac Lane spaces, its effect on $S^{3} \stackrel{i}{\longrightarrow} X_{2 p+2}$ is determined by $i^{*}$. By definition, $y_{1} \in H^{3}(X)$ has the property that $i^{*}\left(y_{1}\right)=\iota$. By condition $(*)$, the other basis elements satisfy $i^{*}\left(y_{i}\right)=0$ for $2 \leqslant i \leqslant r$. For dimensional reasons, $i^{*}\left(x_{2 i_{j}+1}\right)=0$ for $r+1 \leqslant j \leqslant m$. Thus $i$ factors as the composite $S^{3} \hookrightarrow \bar{A}_{1} \hookrightarrow X_{2 p+2}$.

In the case when condition $(*)$ is satisfied, the factorization of $i$ through the homotopy cofibre $\bar{A}$ of $\bar{\alpha}$ immediately implies the following.

Corollary 3.3. If the basis $\mathcal{B}$ for $H^{3}(X)$ satisfies condition (*) then the map $S^{3} \stackrel{i}{\longrightarrow}$ $X$ has the property that $i \circ \bar{\alpha}$ is null homotopic.

Proof of the equivalence of parts (a) and (c) in Theorem 1.1. By Lemma 3.1, we get that part (a) implies part (c). Conversely, suppose that $X$ has a single cell in dimension $3, S^{3} \stackrel{i}{\longrightarrow} X$ is the inclusion of the bottom cell, and $i^{*}(x)=\iota$ for some $x \in H^{3}(X)$ with the property that $\beta \mathcal{P}^{1}(x) \neq 0$. Then the basis $\mathcal{B}$ of $H^{3}(X)$ automatically satisfies condition $(*)$, so by Corollary 3.3 the composite $P^{2 p+1}(p) \stackrel{\bar{\alpha}}{\longrightarrow} S^{3} \stackrel{i}{\longrightarrow} X$ is null homotopic.

\section{The order of $\Omega j\langle 3\rangle$}

By [Th], if $p \geqslant 5$ then $B(3,2 p+1)$ can be given an $H$-structure which is homotopy associative and homotopy commutative, and with respect to this $H$-structure the homotopy fibration $S^{3} \stackrel{s}{\longrightarrow} B(3,2 p+1) \stackrel{q}{\longrightarrow} S^{2 p+1}$ has both $s$ and $q$ being $H$-maps. Further, $B(3,2 p+1)$ satisfies a certain universal property with respect to its $(2 p+1)$ skeleton $A$. Let $\imath: A \longrightarrow B$ be the skeletal inclusion. Note that

$$
H_{*}(B(3,2 p+1)) \cong \Lambda\left(x_{3}, x_{2 p+1}\right) \cong \Lambda\left(\widetilde{H}_{*}(A)\right),
$$

and $\imath$ induces the inclusion of the generating set. In particular, $A$ generates $B$ homologically. The universal property implies a much stronger statement, that $A$ generates $B(3,2 p+1)$ as an $H$-space. To be precise, by $B(3,2 p+1)$ being universal for $A$, we mean that any map $f: A \longrightarrow Z$ into a homotopy associative, homotopy commutative $H$-space $Z$ can be extended to an $H$-map $\bar{f}: B(3,2 p+1) \longrightarrow Z$, and this is the unique $H$-map with the property that $\bar{f} \circ \imath \simeq f$.

Let $m$ be the homotopy associative, homotopy commutative multiplication on $B(3,2 p+1)$. The universal property was used in $[\mathbf{T h}]$ to factor the $p^{t h}$-power map 
on $B(3,2 p+1)$ as

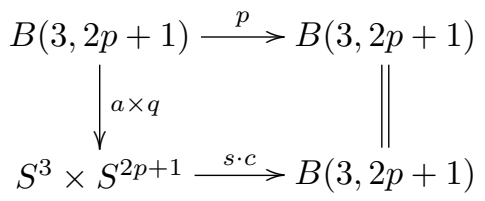

where $a$ is an $H$-map with the property that $a \circ i$ is of degree $p$, and $s \cdot c$ is the composite $S^{3} \times S^{2 p+1} \stackrel{s \times c}{\longrightarrow} B(3,2 p+1) \times B(3,2 p+1) \stackrel{m}{\longrightarrow} B(3,2 p+1)$. In fact, all the maps in (2) are $H$-maps. To see this, we have already mentioned that $a, s$, and $q$ are $H$-maps. By $[\mathbf{G}]$, if $p \geqslant 5$ then any map $S^{2 n+1} \longrightarrow Z$ into a homotopy associative, homotopy commutative $H$-space is an $H$-map. In particular, $c$ is an $H$-map. Since $B(3,2 p+1)$ is a homotopy associative, homotopy commutative $H$-space its multiplication $m$ is an $H$-map, and so both $p$ and $s \cdot c$ are $H$-maps. Thus, part of the strength of (2) is that it is a factorization of the $p^{t h}$-power map through $H$-maps.

Now we refine the factorization in (2) after taking three-connected covers and looping. By Lemma 2.6, $\Omega j\langle 3\rangle \simeq \Omega c \circ t^{\prime}$, where $t^{\prime}=\Omega \delta \circ t$. Thus $\Omega(j\langle 3\rangle \cdot c)$ is homotopic to the composite

$$
\begin{gathered}
\Omega S^{3}\langle 3\rangle \times \Omega S^{2 p+1} \stackrel{t^{\prime} \times 1}{\longrightarrow} \Omega S^{2 p+1} \times \Omega S^{2 p+1} \stackrel{\Omega c \times \Omega c}{\longrightarrow} \Omega B(3,2 p+1)\langle 3\rangle \times \Omega B(3,2 p+1)\langle 3\rangle \\
\stackrel{\Omega m}{\longrightarrow} \Omega B(3,2 p+1)\langle 3\rangle .
\end{gathered}
$$

Since $\Omega c$ is an $H$-map, this is homotopic to the composite

$$
\Omega S^{3}\langle 3\rangle \times \Omega S^{2 p+1} \stackrel{t^{\prime} \times 1}{\longrightarrow} \Omega S^{2 p+1} \times \Omega S^{2 p+1} \stackrel{\mu}{\longrightarrow} \Omega S^{2 p+1} \stackrel{\Omega c}{\longrightarrow} \Omega B(3,2 p+1)\langle 3\rangle
$$

where $\mu$ is the loop multiplication. Note that $S^{2 p+1}$ is an $H$-space so the loop multiplication $\mu$ is homotopic to the loop of the multiplication on $S^{2 p+1}$, implying that $\mu$ is an $H$-map. This lets us reformulate (2) as the following. Let

$$
\theta: \Omega B(3,2 p+1)\langle 3\rangle \longrightarrow \Omega S^{2 p+1}
$$

be the composite $\mu \circ\left(t^{\prime} \times 1\right) \circ(\Omega a\langle 3\rangle \times q)$. Observe that each of the maps in the composite is an $H$-map, and so $\theta$ is an $H$-map.

Proposition 4.1. For $p \geqslant 5$, there is a homotopy commutative square of $H$-maps

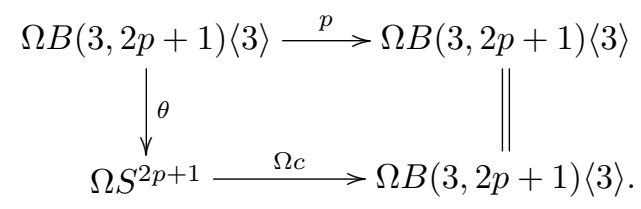

Proof of Theorem 1.4 for $p \geqslant 5$ : Consider the map $\Omega B(3,2 p+1)\langle 3\rangle \stackrel{\Omega j\langle 3\rangle}{\longrightarrow} \Omega X\langle 3\rangle$. As $j$ has the property that $j \circ c$ is null homotopic, the diagram in Proposition 4.1 implies that the composite $\Omega B(3,2 p+1)\langle 3\rangle \stackrel{p}{\longrightarrow} \Omega B(3,2 p+1)\langle 3\rangle \stackrel{\Omega b\langle 3\rangle}{\longrightarrow} \Omega X\langle 3\rangle$ is null homotopic. Since $\Omega b\langle 3\rangle$ is an $H$-map it commutes with power maps, so the composite $p \circ \Omega b\langle 3\rangle$ is null homotopic. That is, $\Omega b\langle 3\rangle$ has order $p$. 
Remark 4.2. Note that the $H$-map property in Proposition 4.1 was not used in the proof of Theorem 1.4. It has been included nevertheless as an interesting feature in its own right which may have applications elsewhere.

The $p=3$ case is different. Observe that the space $B(3,7)$ is homotopy equivalent to the Lie group $S p(2)$ localized at 3 . Since $S p(2)$ is a loop space, it is homotopy associative. By $[\mathbf{M}]$, this loop multiplication is also homotopy commutative at 3 . By $[\mathbf{G r b T}], S p(2)$ at 3 has a limited universal property, but it is unclear whether this property can be used to show the existence of a factorization of the $3^{r d}$-power map on $S p(2)$ through $S^{3} \times S^{5}$ as in (2). So in this case we do without such a factorization and argue differently, but obtain an answer which requires one more loop.

Proof of Theorem 1.4 for $p=3$ : In general, if there is a homotopy pullback

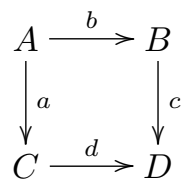

where $D$ is an $H$-space with a homotopy inverse, then there is a homotopy fibration $A \stackrel{a \times b}{\longrightarrow} B \times C \stackrel{c \cdot d^{-1}}{\longrightarrow} D$. In our case, the homotopy pullback in (1) implies that there is a homotopy fibration $S^{7}\{3\} \longrightarrow S^{3}\langle 3\rangle \times S^{5} \stackrel{i\langle 3\rangle \cdot c^{-1}}{\longrightarrow} B(3,7)\langle 3\rangle$. This induces a homotopy fibration

$$
\Omega S^{3}\langle 3\rangle \times \Omega S^{5} \stackrel{\Omega\left(i\langle 3\rangle \cdot c^{-1}\right)}{\longrightarrow} \Omega B(3,7)\langle 3\rangle \stackrel{\partial}{\longrightarrow} S^{7}\{3\}
$$

where $\partial$ is the fibration connecting map. By [N], the $3^{r d}$-power map on $S^{7}\{3\}$ is null homotopic. However, it is not known whether $\partial$ is an $H$-map, so we cannot argue that $3 \circ \partial \simeq \partial \circ 3$ and therefore that $\partial \circ 3$ is null homotopic. However, after looping, this argument does work. We obtain a lift

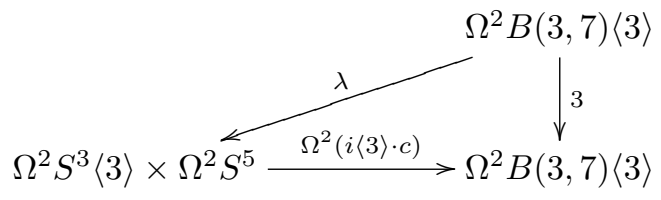

for some map $\lambda$. Since $\Omega^{2} B(3,7)\langle 3\rangle$ is a double loop space, its loop multiplication is an $H$-map. Thus there is a homotopy $\Omega^{2}(i\langle 3\rangle \cdot c) \simeq \Omega^{2} i\langle 3\rangle \cdot \Omega^{2} c$. The remainder of the argument now follows as in the proof of Theorem 1.4 for $p \geqslant 5$, using (3) to replace $(2)$.

\section{References}

[CN] F.R. Cohen and J.A. Neisendorfer, A construction of $p$-local $H$-spaces. Algebraic Topology, Aarhus 1982, 351-359, Lecture Notes in Math. 1051, Springer, Berlin, 1984.

[G] J. Grbić, Universal homotopy associative, homotopy commutative $H$ spaces, PhD Thesis, University of Aberdeen, 2004. 
[GraT] B. Gray and S. Theriault, An elementary construction of Anick's fibration, Geom. Topol. 14 (2010), 243-276.

[GrbT] J. Grbić and S. Theriault, A universal property for $S p(2)$ at 3, Homology, Homotopy, and Appl. 11 (2009), 1-15.

[H] J.R. Harper, $H$-spaces with torsion, Mem. Amer. Math. Soc. 22 (1978), no. 223.

[K] R. Kane, The homology of Hopf spaces, North-Holland Mathematical Library 40, North-Holland Publishing Co, Amsterdam, 1988.

[L] J.P. Lin, Torsion in $H$-spaces II, Ann. of Math. 107 (1978), 41-88.

[M] C.A. McGibbon, Homotopy commutativity in localized groups, Amer. J. Math 106 (1984), 665-687.

[MNT] M. Mimura, G. Nishida, and H. Toda, Mod- $p$ decomposition of compact Lie groups, Publ. RIMS, Kyoto Univ 13 (1977), 627-680.

[N] J.A. Neisendorfer, Properties of certain $H$-spaces, Quart. J. Math. Oxford 34 (1983), 201-209.

[Th] S.D. Theriault, The odd primary $H$-structure of low rank Lie groups and its application to exponents, Trans. Amer. Math. Soc. 359 (2007), 4511-4535.

[To1] H. Toda, On homotopy groups of $S^{3}$-bundles over spheres, J. Math. Kyoto Univ. 2 (1963), 193-207.

[To2] H. Toda, On Iterated Suspensions I, J. Math. Kyoto Univ. 5 (1965), 87-142.

Piotr Beben bebenp@unbc.ca

Stephen Theriault s.theriault@maths.abdn.ac.uk

Department of Mathematical Sciences, University of Aberdeen, Aberdeen AB24 3UE, United Kingdom 